\title{
THz multi line-of-sight polarimeter for fusion reactors
}

\author{
${ }^{1}$ F. Mazzocchi, ${ }^{1}$ G. Grossetti, ${ }^{2}$ A. Mlynek, ${ }^{2}$ E. Poli, ${ }^{1}$ D. Strau $ß$ and ${ }^{1}$ T. Scherer \\ ${ }^{1}$ Karlsruhe Institute of Technology (KIT IAM-AWP), Eggenstein-Leopoldshafen, Germany \\ ${ }^{2}$ Max Planck Institute for Plasma Physics (IPP), Garching, Germany
}

\begin{abstract}
In this paper we present the first preliminary study of a new polarimetry diagnostic system. The device foresees multiple lines of sight, so that the measure of the plasma parameters can be performed at different chords along the poloidal plane, parallel to the equatorial direction, in a single acquisition cycle. Considering the typical plasma conditions (i.e. ASDEX Upgrade) of the actual magnetic confinement machines, we need to employ sources in the range of the low $(<3) \mathrm{THz}$ to have appreciable rotation angles. As source, the diagnostic foresees the use of Quantum Cascade Lasers (QCL) which represents a very promising solution, given their ability to operate at the expected frequency of $1.6 \mathrm{THz}$ at $4.2 \mathrm{~K}$. Since the power of the probe beam is in the order of tenths microwatts, a cryo-detector, such as kinetic inductance detector (KID), is required. This opens the field for a very compact modular machine, composed by a single cryogenic cooler encasing source, detector and the optical section. The assessment study has been performed taking into account the performances, reliability and adaptability to multiple machines, and enriched with estimates of the Faraday rotation at different conditions, using as base data coming from the ASDEX Upgrade (AUG) tokamak located at IPP in Garching.
\end{abstract}

\section{INTRODUCTION}

$\mathrm{I}_{\mathrm{s}}$ $\mathrm{N}$ nuclear fusion research, polarimetry is a wellestablished technique to measure fundamental plasma parameters, such as the electronic density and the poloidal field [1]. By measuring the Faraday rotation angle, the path integral of these two quantities can be obtained. When coupled with the line integrated density measurement along the same chord coming from another instrument (i.e. interferometer), the plasma current density can be estimated. [2]. Innovative calibration methods, such as Complex Amplitude Ratio (CAR) method, can be used to evaluate the line integrated density independently from interferometric data, via the Cotton - Mouton effect induced ellipticity [3]. Additionally, polarimetric data has been also employed in the past in the determination of the q-profiles [4,5]. Finally, an evaluation of the electronic temperature can be obtained by considering the electronic kinetic effects at high temperatures, and a new model for a more precise evaluation in the upcoming ITER has been recently presented [6]. The knowledge of these and other parameters is fundamental to determine the confinement properties of different plasma scenario and ignition criteria fulfillment. By measuring along different cords, this diagnostic is able to characterize the plasma along the whole poloidal plane. In this document, the concepts for a multiple line of sight polarimeter are presented. We present a first assessment on this topic, taking into consideration the state of the art for sources and detectors currently commercially available.

\section{THEORETICAL BACKGROUND}

Polarimetry is the measure of the Faraday rotation angle of an electromagnetic wave when it propagates through a magnetized media (Fig.1). Consider an electromagnetic wave with frequency $v$ propagating into a plasma along the $\mathrm{z}$ direction. The Faraday rotation for small angles is given by [7]

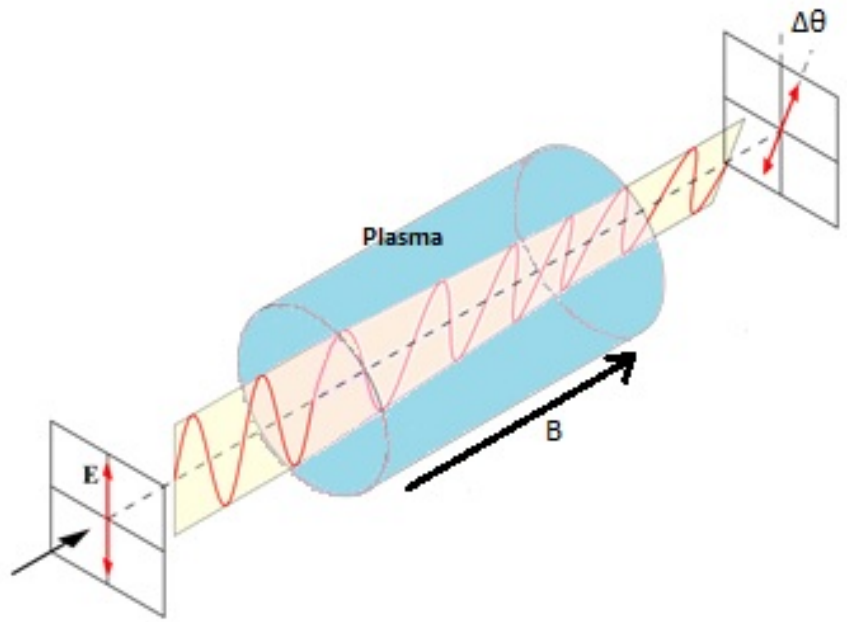

Figure 1 Representation of the Faraday effect

$$
\Delta \theta=\frac{e^{3}}{2 \epsilon_{0} m_{e}^{2} c} \frac{1}{\omega^{2}} \int n_{e}(z) B_{\|}(z) d z
$$

Where $\mathrm{B}_{\|}(\mathrm{z})$ is the longitudinal magnetic field along the line of sight, $\mathrm{n}_{e}$ is the electron density. This formula is valid for non relativistic electron motion 
inside the plasma. The phenomenon arises from the fact that the presence of a magnetic field disrupts the symmetry between the plasma dielectric constant for right and left handed circular polarization components of the original beam, given the different response electrons have to the two components. The result of such asymmetry is the rotation of the polarization vector. The rotation is a function of the inverse square of the frequency, of the electron density and magnetic field. Once coupled with the additional information about the electron density given by an interferometric system, the estimation of both magnetic field and current density can be obtained, given that

$$
\int n_{e}(z) B_{\|}(z) d z \approx J
$$

Current polarimetric systems employ gas lasers, typically HCN [8,9,10]@337 $\mu \mathrm{m}, \mathrm{HCOOH} @ 433 \mu \mathrm{m}$ [11] and DCN@195 $\mu \mathrm{m}[12,13]$, all needing

- large space

- stabilization in both power and wavelengths

- frequent maintenance and gas refill

- safety measures related to the high voltage

The next generations of fusion plants will require more instruments with high reliability, minimal maintenance downtime and a small footprint.

\section{ASSESSMENT STUDY}

The assessment study we performed can be divided into the following steps:

1. Definition of the system specifications, assuming a set of design guidelines. More specifically, the polarimetric system shall have high reliability and availability, simplicity, ease of service and wide angular dynamic range, to maximize the angular resolution and therefore the precision of measurement. Performance - wise, ITER requirements for polarimetric systems have been taken as guidelines. Some of the desired key characteristics include:

- Pump - probe type of input: by monitoring the input power, the device can be equipped with an automatic feedback power control and stabilization system

- Compact: plug and play, ready to measure boxed system

- Reliability and low maintenance
- Multiple line-of-sight

2. Identification of possible solutions: multiple options for both the source and the detector are actually available as state of the art technologies. The assessment of the devices has been carried on under the performances, system specifications and costs points of view

3. Definition of the configuration of the device: polarimeters can be built in a variety of configurations. The parameters that influenced the assessment on the configuration are the same ones used in the devices evaluation

In analogy to the interferometric systems, polarimeters may come in a wide variety of configurations [14]. In an effort to keep the system as simple as possible and to avoid issues deriving from laser stability, vibrations and alignment, the Dodel-Kunz method and a JET-like interferometer - polarimeter where excluded. In order to improve the reliability of the system and minimize maintenance issues, we devised a solution that avoids moving parts, as in the rotating waveplate and dual PEM methods.

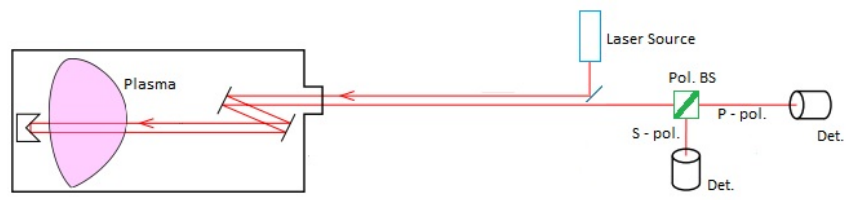

Figure 2 Schematic representation of the double passage dual detector polarimeter

Our iteration of the polarimeter foresees the use of dual detectors, one for each polarization plane of the probe beam (Fig.2). The laser beam enters the plasma, is reflected by retroreflectors placed on the first wall at the high field side, passes through a polarizing beamsplitter, which separates the ordinary and extraordinary components, whose intensities are recorded by the detectors.

Using Quantum Cascade Lasers (QCLs) in place of gas lasers, costs and space footprint can be greatly reduced while power stability and reliability increase. QCLs have a wide frequency range $(0.85 \rightarrow 5 \mathrm{THz}$, roughly $350 \leftarrow 60 \mu \mathrm{m}$ ) with the most common being $2.8 \mathrm{THz}$ $(107 \mu \mathrm{m})$, capable of ambient temperature operations (Fig.3,4). Such a laser would give us rotations in the range of $0-5^{\circ}$ with AUG typical conditions $\left(\mathrm{n}_{\mathrm{e}} 3-\right.$ $9^{*} 10^{19} \mathrm{~m}^{-3}, \mathrm{~B}_{\|} \approx 0.2-0.4 \mathrm{~T}$, plasma radius $\approx 1 \mathrm{~m}$ ), therefore a laser with a lower frequency would be better suited. Prototypes of $1.2 \mathrm{THz}(250 \mu \mathrm{m})$ are being tested and applying a strong magnetic field would decrease the frequency to $0.85 \mathrm{THz}(350 \mu \mathrm{m})$. The problem in this case resides in the fact that, at 
these frequencies, the laser transitions are in the energy range of few $\mathrm{meV}$. At ambient temperature or during $\mathrm{CW}$ operation, the raise in thermal activity of the device can lead to longitudinal optical phonons scattering between lasing levels, resulting in a degradation of the population inversion. These devices therefore require cooling at liquid helium temperature levels in order to work properly $[15,16]$.

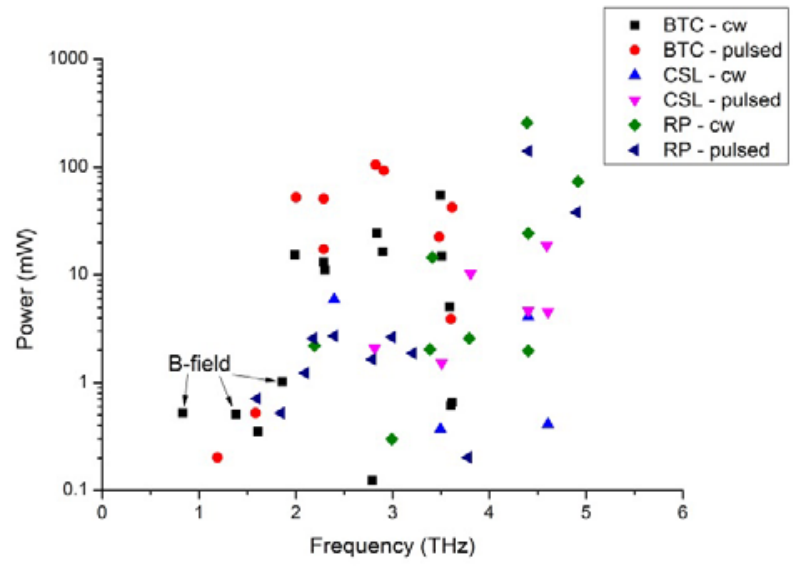

Figure 3 Peak optical power as a function of lasing frequency for different kind QCLs, based on active region design $(C S L=$ Chirped SuperLattice, BTC $=$ Bound - To - Continuum, RP = Resonant Photon) [13]

Ideally, a QCL laser source for a double pass configuration would be characterized by a wavelength around $200 \mu \mathrm{m}(1.5 / 1.6 \mathrm{THz})$ and able to emit 100 $\mathrm{mW}$ power level at temperatures in the order of $250 \mathrm{~K}$, enabling the use of Peltier cells as cooling system.

The current state of the art for QCLs at $1.6 \mathrm{THz}$ sits around $0.3 \mathrm{~mW}$ for the maximum power extracted at liquid ${ }^{4} \mathrm{He}$ temperatures [17]. The system will therefore require a cryostat, in the form of a pulse tube refrigerator, given that as per ITER specifications He bath cryocoolers are to be avoided.

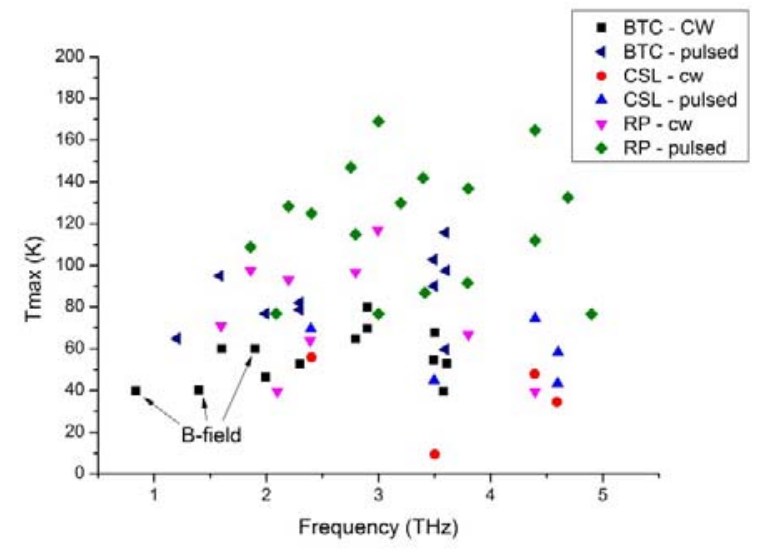

Figure 4 Peak operating temperatures vs lasing frequency for QCLs[13]
The inherent advantages of QCLs are their extremely compact size, ruggedness, and much cheaper price. They require minimal maintenance and the sources can be easily swapped in case of need. Their cheap price allows also to design multiple sources machines for multiple lines of sight at a fraction of the cost of a single gas laser operating at $\mathrm{THz}$ frequencies.

Whereas the gas lasers classically employed in polarimetric diagnostics have powers in the order of hundreds of milliwatts [10], commercially available QCL emitting at analogous wavelengths scale down to tenths of milliwatts. The polarimeter must be therefore equipped with suitable high sensitivity detectors to account for the decreased intensity of the probing beam. Developed for the use in astrophysics and astronomy $([18,19,20,21])$ where power available is in the order of the nano and picowatt, Kinetic Inductance Detectors represent a viable solution for applications in plasma diagnostics. In such detectors photons with energy higher than the gap energy of an absorbing superconductor break Cooper pairs and change the absorber macroscopic electrical properties. The increased density of quasi-particles (conducting electrons) changes the surface reactance of the superconductor (kinetic inductance effect). This effect can be measured efficiently when the absorber is implemented into a superconductor resonator circuit coupled to a transmission line, via the complex transmission scattering parameter S21. The Al KID reaches a NEP of around $10^{-18} \mathrm{~W} \cdot \mathrm{Hz}^{-1 / 2}$, but requires temperatures in the order of 250-300 $\mathrm{mK}$ [22], increasing both the assembly and operating costs. The usage of high $T_{c}$ materials in the construction of these devices can, nevertheless, solve this problem. Niobium Nitride $(\mathrm{NbN})$ is the material of choice for the production of the KIDs. With a critical temperature around $16 \mathrm{~K}$ [23], these thin films transit to the superconducting state at a temperature higher than liquid $\mathrm{He}(4.2 \mathrm{~K})$, simplifying the characterization of the final product and its operation. The higher critical temperature of the $\mathrm{NbN}$ films will determine a decrease of the NEP, to be determined once the first detector prototypes are produced. Production, characterization and testing of the KIDs will represent the biggest part of the project related to the realization of the polarimeter described in this paper.

Once the two polarizations components are recorded by the detectors, the Faraday angle is given by the arc tangent of the amplitude ratio of the two signals.

\section{VALIDATION OF THE FIRST ASSESSMENT}

In order to validate the concepts we exposed so far, we ran some calculations using as benchmark reference 
the values obtained from actual measurements made at ASDEX Upgrade. The system located at Garching is based on a DCN Laser emitting radiation@195 $\mu \mathrm{m}$. A beam from the DCN system passing through the plasma above the midplane at AUG high density ( $9^{*} 10^{19}-10^{20} \mathrm{~m}^{-3}$ ) should have a rotation of the polarization vector of roughly 10 degrees. Two different density profiles (low $\sim 3 * 10^{19} \mathrm{~m}^{-3} \mathrm{MAX}$ and high $\sim 9 * 10^{19} \mathrm{~m}^{-3}$ MAX, Fig.5) where provided by the AUG team,

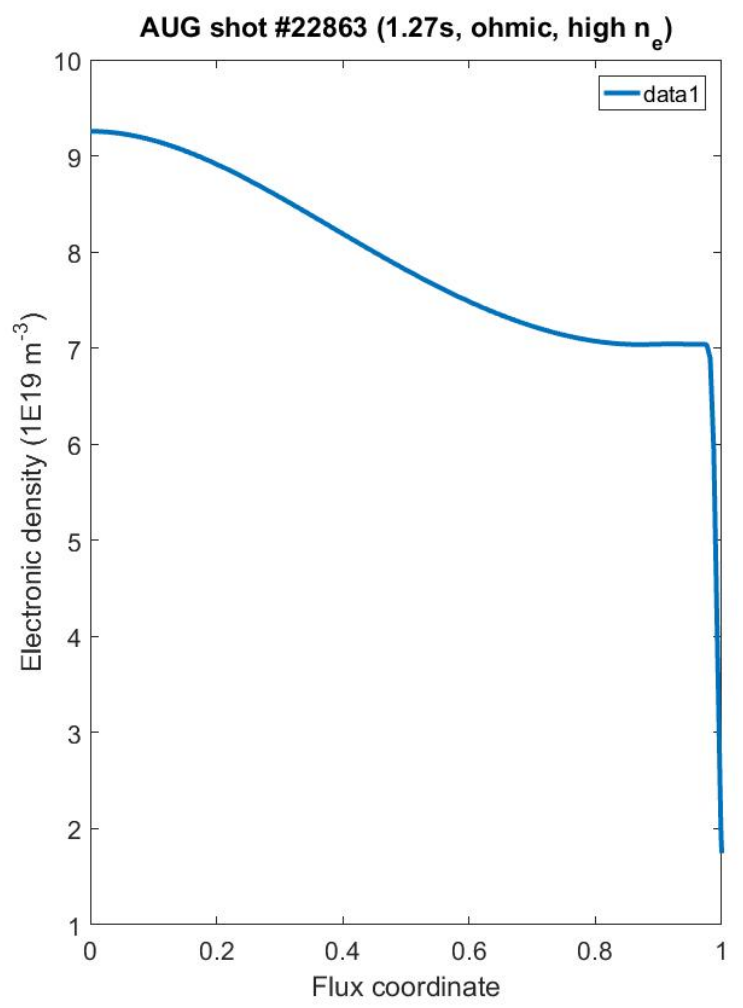

Figure 5 AUG "high" density profile

while a third (medium density) was obtained by averaging the values in the density matrices. Magnetic equilibria (Fig.6) were also provided, completing the datasets necessary to run the calculations via MATLAB script which performs the numerical integration of Eq. (1).

Three different probe beam frequencies were simulated at $1.3,1.6$ and $1.8 \mathrm{THz}$, corresponding to $230.6,187.3$ and $166.5 \mu \mathrm{m}$ wavelength. The results are summarized in Table 1. The results are in accordance with our cross reference value of 10 degrees for the AUG DCN polarimeter. Taking into account the considerations made about the dynamic range of the device, the low density regime, where the maximum value is around $3 * 10^{19} \mathrm{~m}^{-3}$ is the lower applicability limit for the two higher frequency sources.
If we are to consider possible applications of the device to machines with higher magnetic fields and larger dimensions than AUG, the $1.3 \mathrm{THz}$ beam can be subject to rotations over $+/-90^{\circ}$, requiring complicate phase analysis techniques to discern between partial and complete rotations. $1.6 \mathrm{THz}$ is our candidate frequency for this application (Fig.7). In any case the modularity of the instrument and the size of QCLs allow for easy swapping of the sources to accommodate the operational conditions of the target plasma.

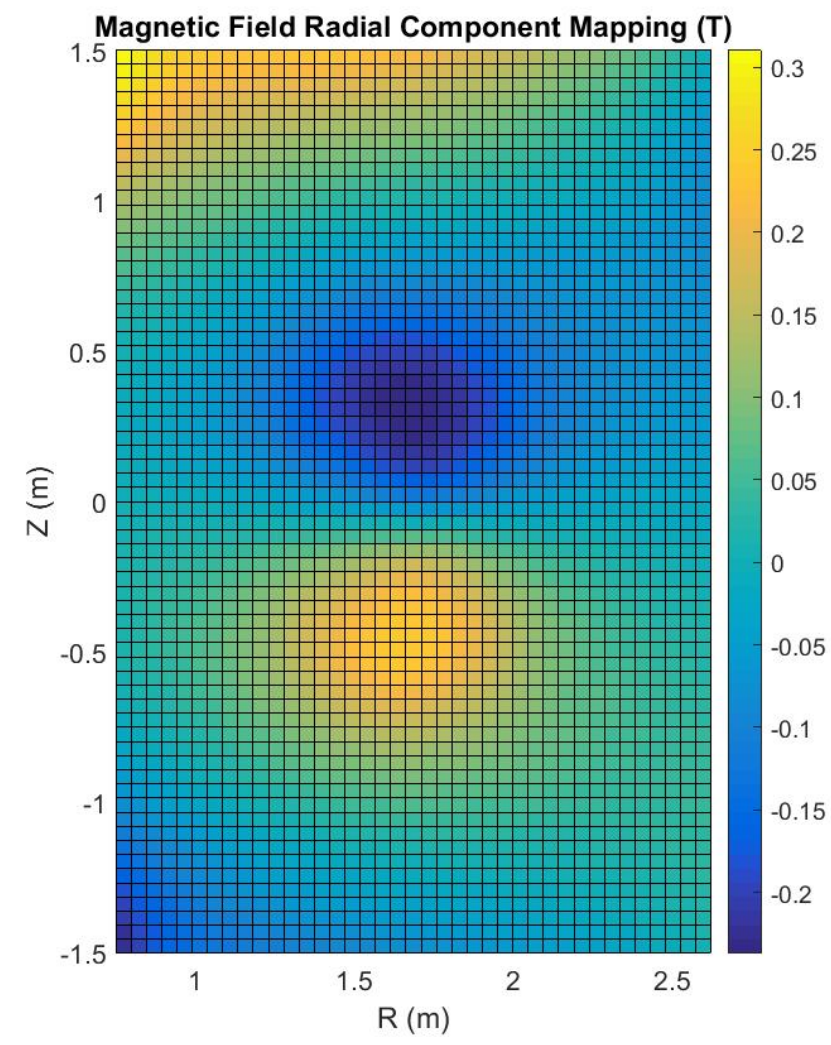

Figure 6 AUG magnetic field (radial component) equilibria

\begin{tabular}{|l|c|c|c|}
\hline & Low Dens. & Mid. Dens. & High Dens. \\
\hline $\begin{array}{l}1.3 \mathrm{THz} \\
(230 \mu \mathrm{m})\end{array}$ & $6.5 /-7.3$ & $13 /-14$ & $19.6 /-22$ \\
\hline $\begin{array}{l}1.6 \mathrm{THz} \\
(187 \mu \mathrm{m})\end{array}$ & $4.3 /-4.8$ & $8.6 /-9.7$ & $12.9 /-14.6$ \\
\hline $\begin{array}{l}1.8 \mathrm{THz} \\
(166 \mu \mathrm{m})\end{array}$ & $3.4 /-3.8$ & $6.8 /-7.7$ & $10.2 /-11.5$ \\
\hline
\end{tabular}

Table.1 Rotation values (in degs) for the Faraday effects, at the maximum field points 


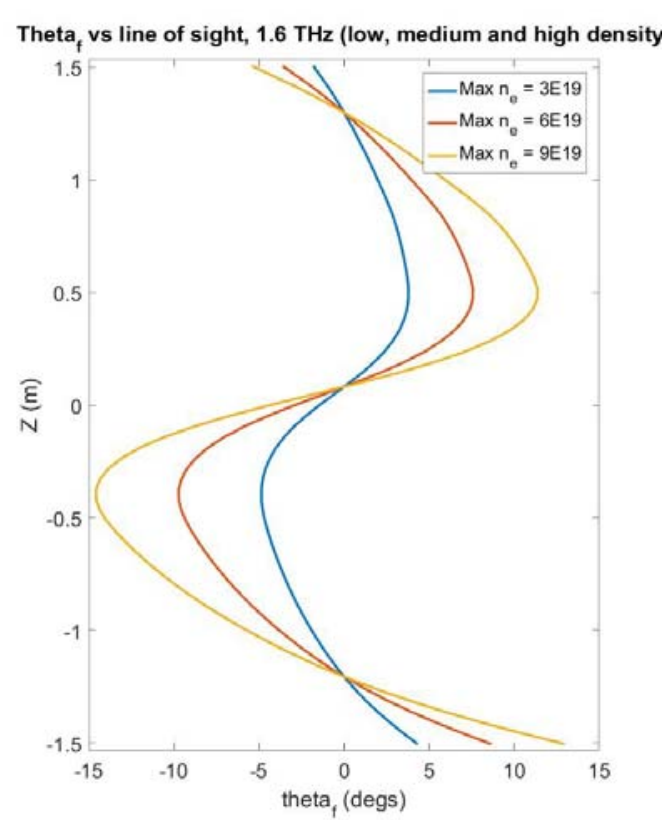

Figure 7 Faraday rotation angle, calculated at the three density profiles for a $1.6 \mathrm{THz}$ probe beam

\section{Future Study REFinements}

The calculations presented above are just a first step into a more refined assessment to be carried out in the next future. Improvements will include

- Re-modelization of the Faraday rotation using solutions to the Stokes equations, as presented in $[24,25,26]$

- Evaluation of the impact of Cotton - Mouton and electronic kinetic effects over the polarization measurements for data correction [3]

- Better definition of the required optical system and modelling for calibration purposes [3]

- Evaluation of the applicability of the final instrument to temperature measurements and q-profile extrapolation

\section{SUMMARY}

We presented a first assessment about a possible innovative solution for polarimetric measurements in the future fusion reactors. The rationale takes into account key properties such has compact size, low maintenance, low cost, stability, flexibility and robustness, with multiple line of sight. The combination of devices that best suites our needs is a cryogenic system, based on a pulsed tube refrigerator containing the QCL source @1.6 THz, optics and the detectors. A first set of calculations were performed, to validate the applicability of the concepts exposed, returning promising results for the device to be. A more refined assessments that takes into consideration a better model and additional phenomena occurring to the beam in the plasma is foreseen for the immediate future.

\section{REFERENCES}

[1] W Kunz and TFR Equipe. First measurement of poloidal-field-induced faraday rotation in a tokamak plasma. Nuclear Fusion, 18(12):1729, 1978.

[2] H Soltwisch. Current density measurements in tokamak devices. Plasma physics and controlled fusion, 34(12):1669, 1992.

[3] A. Boboc, B. Bieg, R. Felton, S. Dalley and Y. Kravtsov, A novel calibration method for the JET real-time far infrared polarimeter and integration of polarimetry-based lineintegrated density measurements for machine protection of a fusion plant (invited), Review Of Scientific Instruments 86(7): 091301, 2015

[4] H R Koslowski and H Soltwisch, Electron density and $q$ profile measurements with the far-IR interferometer polarimeter on the TEXTOR tokamak, Fusion Engineering and Design 34-35 (3):143, 1997

[5] L Zabeo, A Murari, E, Joffrin, D. Mazon and C. Taliercio, A versatile method for the real time determination of safety factor and density profiles in JET, Plasma physics and controlled fusion, 44(10):2483, 2002.

[6] V V Mirnov, D L Brower, D J Hartog, W X Ding, J Duff and E. Parke, Electron kinetic effects on interferometry, polarimetry, and Thomson scattering measurements in burning plasmas, Review of scientific instruments 85(8):11D302, 2014

[7] S Mancuso and S R Spangler. Faraday rotation and models for the plasma structure of the solar corona. The Astrophysical Journal, 539(1):480, 2000.

[8] P Belland, D Veron, and LB Whitbourn. Mode study, beam characteristics and output power of a cw $377 \mu \mathrm{m} \mathrm{HCN}$ waveguide laser. Journal of Physics D: Applied Physics, (18):2113, 1975.

[9] M Kawamura, I Okabayashi, and T Fukuyama. A capacitively coupled rf- excited cw-HCN laser. IEEE journal of quantum electronics, 21(11):1833-1837, 1985.

[10] JB Zhang, XC Wei, HQ Liu, JJ Shen, L Zeng, and YX Jie, Output power stability of an HCN laser using a stepping motor for the east interferometer system. Journal of Instrumentation, 10(11):C11004, 2015. 
[11] YG Li, Y Zhou, Y Li, HX Wang, ZC Deng, J Yi, XQ $\mathrm{Ji}$, et al. First electron density fluctuation measurement via the $\mathrm{HCOOH}$ laser far-forward collective scattering on HL2A tokamak. Journal of Instrumentation, 11(02):C02002, 2016.

[121] A Mlynek, L Casali, O Ford, H Eixenberger, and ASDEX Upgrade Team. Fringe jump analysis and implementation of polarimetry on the ASDEX Upgrade DCN interferometer. Review of Scientific Instruments, 85(11):11D408, 2014.

[13] G Braithwaite, N Gottardi, G Magyar, J O'Rourke, J Ryan, and D Veron. Jet polari-interferometer. Review of scientific instruments, 60(9):2825-2834, 1989.

[14] R Imazawa, Y Kawano, T Akiyama, K Nakayama, and K Itami. Polarization measurement techniques suitable for ITER poloidal polarimeter. In Proceedings of the $41 \mathrm{st}$ Plasma Physics Conference on Plasma Physics (EPS2014)(IOP, Berlin, Germany, 2014) P, vol. 5, 2014.

[15] BS Williams. Terahertz quantum-cascade lasers. Nature photonics, 1(9):517-525, 2007.

[16] H Li, JC Cao, YJ Han, ZY Tan, and XG Guo. Temperature profile modelling and experimental investigation of thermal resistance of terahertz quantumcascade lasers. Journal of Physics D: Applied Physics, 42(20):205102, 2009.

[17] C. Walther, M. Fischer, G. Scalari, R. Terazzi, N. Hoyler, and J. Faist, "Quantum cascade lasers operating from 1.2 to 1.6 THz," Applied Physics Letters, vol. 91, no. 13, p. 131122, 2007.

[18] G Vardulakis, S Withington, DJ Goldie, and DM Glowacka. Superconducting kinetic inductance detectors for astrophysics. Measurement Science and Technology, 19(1):015509, 2007.

[19] A Monfardini, A Benoit, A Bideaud, L Swenson, A Cruciani, P Camus, C Hoffmann, FX Désert, S Doyle, P Ade, et al. A dual-band millimeter-wave kinetic inductance camera for the IRAM $30 \mathrm{~m}$ telescope. The Astrophysical Journal Supplement Series, 194(2):24, 2011.

[20] J Zmuidzinas and P L Richards. Superconducting detectors and mixers for millimetre and submillimetre astrophysics. Proceedings of the IEEE, 92(10):1597-1616, 2004.

[21] J Schlaerth, A Vayonakis, P Day, J Glenn, J Gao, S Golwala, S Kumar, H LeDuc, B Mazin, J Vaillancourt, et al. A millimeter and submillimeter kinetic inductance detector camera. Journal of Low Temperature Physics, 151(3):684689, 2008.
[22] J Baselmans, SJC Yates, R Barends, YJY Lankwarden, JR Gao, H Hoevers, and TM Klapwijk. Noise and sensitivity of aluminum kinetic inductance detectors for sub-mm astronomy. Journal of Low Temperature Physics, 151(12):524-529, 2008.

[23] YM Shy, LE Toth, and R Somasundaram. Superconducting properties, electrical resistivities, and structure of $\mathrm{NbN}$ thin films. Journal of Applied Physics, 44(12):5539-5545, 1973.

[24] S E Segre, A review of plasma polarimetry - theory and methods, Plasma physics and controlled fusion 41(2):57, 1999

[25] F P Orsitto, A Boboc, C Mazzotta, E Giovannozzi, L Zabeo and JET EFDA Contributors, Modelling of polarimetry measurements at JET, Plasma Physics and Controlled Fusion 50(10):115009, 2008

[26] F P Orsitto, A Boboc, P Gaudio, M Gelfus, E Giovannozzi, C Mazzotta, A Murari and JET EFDA Contributors, Analysis of Faraday rotation in JET polarimetric measurements, Plasma Physics and Controlled Fusion 53(1):035001, 2011 